\title{
Correction to: CpG-creating mutations are costly in many human viruses
}

Victoria R. Caudill', ${ }^{1,2}$ Sarina Qin ${ }^{1,3} \cdot$ Ryan Winstead ${ }^{1}$. Jasmeen Kaur ${ }^{1}$.

Kaho Tisthammer ${ }^{1}$. E. Geo Pineda ${ }^{1}$. Caroline Solis ${ }^{1}$. Sarah Cobey ${ }^{16}$. Trevor Bedford ${ }^{4}$. Oana Carja ${ }^{5} \cdot$ Rosalind M. Eggo $^{6} \cdot$ Katia Koelle $^{7} \cdot$ Katrina Lythgoe $^{8} \cdot$ Roland Regoes $^{17}$. Scott Roy ${ }^{1} \cdot$ Nicole Allen $^{1}$. Milo Aviles ${ }^{1}$ - Brittany A. Baker ${ }^{1}$. William Bauer ${ }^{1}$. Shannel Bermudez ${ }^{1}$. Corey Carlson ${ }^{1} \cdot$ Edgar Castellanos $^{1}$ • Francisca L. Catalan ${ }^{1,9}$. Angeline Katia Chemel ${ }^{1}$ - Jacob Elliot ${ }^{1}$ - Dwayne Evans ${ }^{1,10}$. Natalie Fiutek ${ }^{1}$. Emily Fryer ${ }^{1,11}$. Samuel Melvin Goodfellow ${ }^{1,12} \cdot$ Mordecai Hecht $^{1} \cdot$ Kellen Hopp $^{1}$. E. Deshawn Hopson Jr. ${ }^{1}$. Amirhossein Jaberi ${ }^{1}$. Jennifer Kim ${ }^{1}$. Christen Kinney ${ }^{1}$. Derek Lao ${ }^{1}$ - Adrienne Le ${ }^{1}$. Jacky Lo ${ }^{1}$ - Alejandro G. Lopez ${ }^{1}$. Andrea López ${ }^{1}$. Fernando G. Lorenzo ${ }^{1}$. Gordon T. Luu ${ }^{1}$ Andrew R. Mahoney ${ }^{1} \cdot$ Rebecca L. Melton $^{1,13}$. Gabriela Do Nascimento ${ }^{1}$ - Anjani Pradhananga ${ }^{1}$. Nicole S. Rodrigues ${ }^{1,14}$. Annie Shieh ${ }^{1} \cdot$ Jasmine Sims $^{1,15} \cdot$ Rima Singh $^{1} \cdot$ Hasan Sulaeman $^{1} \cdot$ Ricky Thu $^{1}$. Krystal Tran ${ }^{1} \cdot$ Livia Tran $^{1} \cdot$ Elizabeth J. Winters ${ }^{1} \cdot$ Albert Wong $^{1} \cdot$ Pleuni S. Pennings ${ }^{1}$ (D)

\section{Correction to: Evolutionary Ecology https://doi.org/10.1007/s10682-020-10039-z}

In the original article, the co-author name "Jennifer Kim" has been inadvertently missed during the publication process. The complete author group is given in this correction.

Publisher's Note Springer Nature remains neutral with regard to jurisdictional claims in published maps and institutional affiliations.

\section{Affiliations}

Victoria R. Caudill ${ }^{1,2}$. Sarina Qin ${ }^{1,3} \cdot$ Ryan Winstead $^{1}$. Jasmeen Kaur ${ }^{1}$. Kaho Tisthammer ${ }^{1}$. E. Geo Pineda ${ }^{1}$. Caroline Solis ${ }^{1}$. Sarah Cobey ${ }^{16} \cdot$ Trevor Bedford $^{4}$. Oana Carja ${ }^{5} \cdot$ Rosalind M. Eggo $^{6} \cdot$ Katia Koelle $^{7} \cdot$ Katrina Lythgoe $^{8} \cdot$ Roland Regoes $^{17}$. Scott Roy ${ }^{1} \cdot$ Nicole Allen $^{1}$ - Milo Aviles ${ }^{1}$ - Brittany A. Baker ${ }^{1}$ - William Bauer ${ }^{1}$. Shannel Bermudez ${ }^{1}$. Corey Carlson ${ }^{1} \cdot$ Edgar Castellanos $^{1} \cdot$ Francisca L. Catalan $^{1,9}$.

The original article can be found online at https://doi.org/10.1007/s10682-020-10039-z.

Pleuni S. Pennings pennings@sfsu.edu

Extended author information available on the last page of the article 
Angeline Katia Chemel ${ }^{1}$. Jacob Elliot ${ }^{1} \cdot$ Dwayne Evans $^{1,10} \cdot$ Natalie Fiutek $^{1}$. Emily Fryer ${ }^{1,11}$. Samuel Melvin Goodfellow ${ }^{1,12} \cdot$ Mordecai Hecht $^{1} \cdot$ Kellen Hopp $^{1}$. E. Deshawn Hopson Jr. ${ }^{1}$. Amirhossein Jaberi ${ }^{1}$. Jennifer Kim ${ }^{1}$. Christen Kinney ${ }^{1}$. Derek Lao ${ }^{1}$. Adrienne Le ${ }^{1}$. Jacky Lo ${ }^{1}$ - Alejandro G. Lopez ${ }^{1}$. Andrea López ${ }^{1}$. Fernando G. Lorenzo ${ }^{1}$. Gordon T. Luu ${ }^{1}$. Andrew R. Mahoney ${ }^{1} \cdot$ Rebecca L. Melton $^{1,13}$. Gabriela Do Nascimento ${ }^{1}$. Anjani Pradhananga ${ }^{1}$. Nicole S. Rodrigues, ${ }^{1,14}$. Annie Shieh ${ }^{1} \cdot$ Jasmine Sims ${ }^{1,15} \cdot$ Rima Singh $^{1} \cdot$ Hasan Sulaeman $^{1} \cdot$ Ricky Thu $^{1}$. Krystal $\operatorname{Tran}^{1} \cdot$ Livia Tran $^{1} \cdot$ Elizabeth J. Winters ${ }^{1} \cdot$ Albert Wong $^{1} \cdot$ Pleuni S. Pennings $^{1}$ (D)

Sarah Cobey

cobey@uchicago.edu

Roland Regoes

roland.regoes@env.ethz.ch

Department of Biology, San Francisco State University, San Francisco, CA, USA

Department of Biology, University of Oregon, Eugene, OR, USA

3 Quantitative Systems Biology, Univeristy of California, Merced, CA, USA

4 Vaccine and Infectious Disease Division, Fred Hutchinson Cancer Research Center, Seattle, WA, USA

5 Department of Computational Biology, School of Computer Science, Carnegie Mellon University, Pittsburgh, USA

6 Department of Infectious Disease Epidemiology, London School of Hygiene \& Tropical Medicine, London, UK

7 Department of Biology, Emory University, Atlanta, GA, USA

8 Big Data Institute, University of Oxford, Oxford, UK

9 Department of Neurological Surgery, University of California, San Francisco, CA, USA Department of Organismic and Evolutionary Biology, Harvard University, Cambridge, MA, USA Biochemistry, Molecular, Cellular and Developmental Biology Graduate Group, University of California, Davis, CA, USA 